\title{
Full-length transcriptome sequences of Agropyron cristatum facilitate the prediction of putative genes for thousand-grain weight in a wheat- $A$. cristatum translocation line
}

Shenghui Zhou', Jinpeng Zhang ${ }^{1}$, Haiming Han ${ }^{1}$, Jing Zhang ${ }^{2}$, Huihui Ma' ${ }^{1}$ Zhi Zhang ${ }^{1}$, Yuqing Lu', Weihua Liu', Xinming Yang ${ }^{1}$, Xiuquan $\mathrm{Li}^{1}$ and Lihui $\mathrm{Li}^{\mathrm{i}^{*}}$ (1)

\begin{abstract}
Background: Agropyron cristatum (L.) Gaertn. ( $2 n=4 x=28$; genomes PPPP) is a wild relative of common wheat (Triticum aestivum L.) and provides many desirable genetic resources for wheat improvement. However, there is still a lack of reference genome and transcriptome information for A. cristatum, which severely impedes functional and molecular breeding studies.

Results: Single-molecule long-read sequencing technology from Pacific Biosciences (PacBio) was used to sequence full-length cDNA from a mixture of leaves, roots, stems and caryopses and constructed the first full-length transcriptome dataset of A. cristatum, which comprised 44,372 transcripts. As expected, the PacBio transcripts were generally longer and more complete than the transcripts assembled via the Illumina sequencing platform in previous studies. By analyzing RNA-Seq data, we identified tissue-enriched transcripts and assessed their GO term enrichment; the results indicated that tissue-enriched transcripts were enriched for particular molecular functions that varied by tissue. We identified 3398 novel and 1352 A. cristatum-specific transcripts compared with the wheat gene model set. To better apply this A. cristatum transcriptome, the $A$. cristatum transcripts were integrated with the wheat genome as a reference sequence to try to identify candidate A. cristatum transcripts associated with thousand-grain weight in a wheat-A. cristatum translocation line, Pubing 3035 .

Conclusions: Full-length transcriptome sequences were used in our study. The present study not only provides comprehensive transcriptomic insights and information for A. cristatum but also proposes a new method for exploring the functional genes of wheat relatives under a wheat genetic background. The sequence data have been deposited in the NCBI under BioProject accession number PRJNA534411.
\end{abstract}

Keywords: Full-length transcriptome, Wheat, Wild relative, Agropyron cristatum, Gene expression, Thousand-grain weight

* Correspondence: lilihui@caas.cn

${ }^{1}$ National Key Facility for Crop Gene Resources and Genetic Improvement, Institute of Crop Sciences, Chinese Academy of Agricultural Sciences, Beijing 100081, China

Full list of author information is available at the end of the article

(c) The Author(s). 2019 Open Access This article is distributed under the terms of the Creative Commons Attribution 4.0 International License (http://creativecommons.org/licenses/by/4.0/), which permits unrestricted use, distribution, and reproduction in any medium, provided you give appropriate credit to the original author(s) and the source, provide a link to the Creative Commons license, and indicate if changes were made. The Creative Commons Public Domain Dedication waiver (http://creativecommons.org/publicdomain/zero/1.0/) applies to the data made available in this article, unless otherwise stated. 


\section{Background}

As the most widely cultivated crop on Earth, wheat (Triticum aestivum L., $2 \mathrm{n}=6 \mathrm{x}=42$, genomes AABBDD) contributes approximately a fifth of the total calories consumed by humans and provides more protein than any other food source [1]. However, due to historical artificial selection and domestication, the genetic diversity of modern wheat is relatively narrow, which is one of the bottlenecks for breakthroughs in wheat improvement [2-4]. Natural variation from collections of wild wheat relatives has been and remains an important facilitator of wheat genetic advances, since these relatives conserve considerable genetic variability of adaptive traits that can be transferred via artificially innovated introgression lines by direct hybridization [5-9].

The genus Agropyron Gaertn., called the crested wheatgrass complex, is an out-crossing tertiary gene pool relative of wheat and built upon one basic $\mathrm{P}$ genome with 3 ploidy levels [10]. The tetraploid crested wheatgrass Agropyron cristatum (L.) Gaertn. ( $2 \mathrm{n}=4 \mathrm{x}=28$, genome PPPP) not only provides protein as a forage source but also possesses several desirable traits for wheat improvement. In the early 1990 s, several wheat- $A$. cristatum derivative lines were produced via the intergeneric hybridization of wheat cv. Fukuhokumugi (Fukuho) and A. cristatum accession Z559 and embryo rescue [11]. Several of these lines, including additional lines, disomic substitution lines, translocation lines and introgression lines, exhibit potentially valuable traits for wheat improvement, such as disease resistance, abiotic and biotic stress tolerance and high yield, and these lines have therefore been used in wheat-breeding programmes [1215]. Among these lines, Pubing 3035, a Ti1AS-6PL1AS.1AL intercalary translocation, was derived from the offspring of a wheat $A$. cristatum $6 \mathrm{P}$ chromosome addition line; notably, the 6P chromosomal segment played an important role in regulating the thousand-grain weight and spike length [15]. Although the growth characteristics and utilization of wheat- $A$. cristatum derivative lines in wheatbreeding programmes have been extensively investigated, little is known regarding the nature of the gene and the mechanism by which it confers superior traits.

As a result of the low frequency of pairing and suppressed recombination between the genomes of wild wheat relatives and wheat, it is extremely difficult to characterize genes from wheat wild relatives through a map-based cloning strategy under a wheat genetic background. Comprehensive approaches, including cytogenetic stock development, mutagenesis, resistance gene enrichment and sequencing-Pacific Biosciences (PacBio), long-range assembly, and functional analysis, were successively used to successfully clone the $P m 21$ gene, which confers high resistance to Blumeria graminis f. sp. tritici (Bgt) in wheat throughout all growth stages, from the wild species Haynaldia villosa [16]. At the same time, Pm21 was also isolated and functionally validated via the discovery of Bgt-susceptible Dasypyrum villosum resources and construction of a genetic population using resistant intervals [17]. Placido and colleagues identified candidate genes associated with root development from the wheat-Agropyron elongatum translocation line by transcriptome analysis, but the relationship between these candidate genes and improved drought adaptation has not yet been elucidated [18]. Most of the studies related to the gene cloning of wild relatives have focused on disease resistance genes, but no relevant studies have reported the cloning of genes associated with complex traits, such as yield-related traits in derived lines. The lack of reference genome sequences severely impedes in-depth molecular breeding and gene functional studies of important wheat wild relatives. Therefore, to reveal the genetic bases of important traits and understand their molecular mechanistic bases, it is particularly urgent to develop an effective strategy for excavating functional candidate genes from wheat and wild relative-derived germplasms expressing superior traits.

RNA-sequencing (RNA-Seq) has recently become a popular technique because it is cost-effective, and it does not rely on a reference genome [19]. RNA-Seq of $A$. cristatum Z559 by the Illumina platform has enabled the successful annotation of orthologous genes related to multiple agronomic traits in A. cristatum [20] and has provided many new insights into the phylogenetic relationship and interspecific variation between $A$. cristatum and wheat [21]. However, the short sequencing reads of the Illumina platform make the assembly and annotation of the A. cristatum transcriptome incomplete and error-prone. Recently, single-molecule, real-time (SMRT) sequencing technology from PacBio has provided an efficient approach to sequence full-length (FL) cDNA molecules and has been successfully used for whole-transcriptome profiling in many animal and plant species [22-34]. Compared with Illumina and other second-generation sequencing techniques, the advantages of PacBio transcriptome sequencing not only allow complete cDNA sequences containing both the $5^{\prime}$ and $3^{\prime}$ ends to be obtained but also enable identification of alternative isoforms $[25,26]$.

In this study, we present the first report on the singlemolecule FL sequencing, annotation and expression of the A. cristatum Z559 transcriptome and the application of this transcriptome in the identification of candidate alien genes associated with thousand-grain weight in the wheat- $A$. cristatum translocation Pubing 3035 (Fig. 1). Single-molecule long-read transcriptome sequencing of A. cristatum Z559 was performed using the PacBio Sequel platform, and full-length, non-concatemer (FLNC) transcripts were constructed and annotated. Tissuespecific FLNC transcripts were revealed in A. cristatum using RNA-Seq. Then, novel and A. cristatum-specific transcripts were identified by comparison with the wheat 


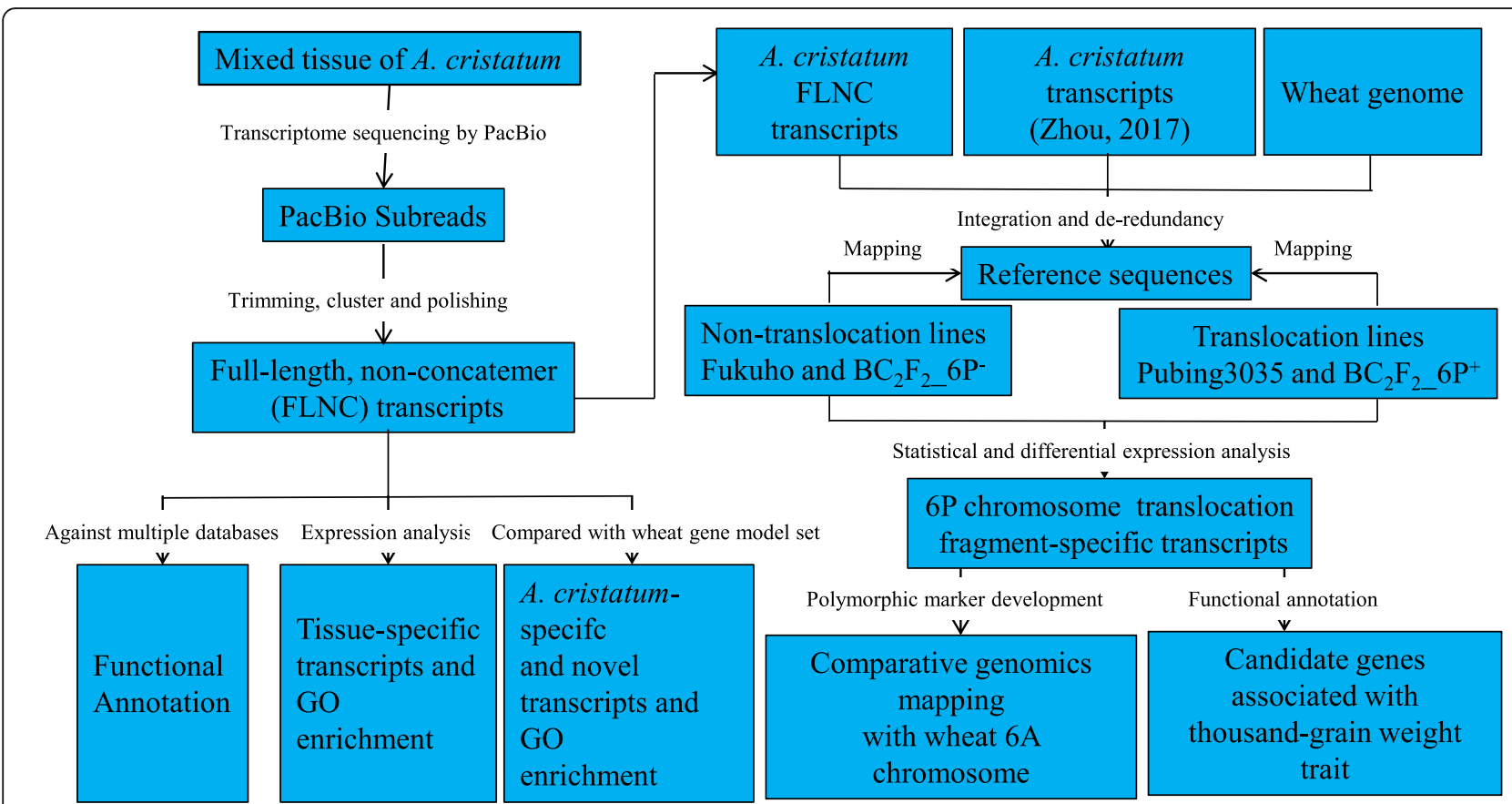

Fig. 1 Pipeline for constructing the A. cristatum transcriptome and the application of this transcriptome in the identification of candidate alien genes in wheat-A. cristatum translocation line Pubing 3035

gene model set. Furthermore, by integrating the A. cristatum transcripts, including FLNCs and transcripts assembled in previous studies [21], and the wheat genome as reference sequences, candidate $A$. cristatum transcripts associated with thousand-grain weight were identified in Pubing 3035. The present study not only provides comprehensive transcriptomic insights and information for $A$. cristatum but also proposes a new method for the exploration of functional genes from wheat relatives under a wheat genetic background.

\section{Methods}

\section{Plant materials}

The A. cristatum accession Z559 $(2 \mathrm{n}=4 \mathrm{x}=28$, PPPP, from Xinjiang, China), a representative tetraploid A. cristatum, has been previously described [20] and cultivated in the experimental field of the Chinese Academy of Agricultural Sciences, Beijing, China (E116.33, N39.96). Fukuho, translocation line Pubing 3035 and their $\mathrm{BC}_{2} \mathrm{~F}_{2}$ population, which was produced with the recurrent parent Fukuho, were planted in the experimental field of the Chinese Academy of Agricultural Sciences, Xinxiang, Henan province, China (E113.46, N35.8).

\section{Tissue sampling and RNA isolation}

Leaves, stems, roots and caryopses (growth stage 54) from A. cristatum plants, leaves and caryopses (growth stage 54, 73, 75 and 77), from Fukuho, Pubing 3035 and their $\mathrm{BC}_{2} \mathrm{~F}_{2}$ population, were collected [35]. The samples of $A$. cristatum, Fukuho and Pubing 3035 consisted of tissues from 5 different plants. According to the presence of the translocation fragment, as determined by molecular makers developed by Zhang et al. [14], the $\mathrm{BC}_{2} \mathrm{~F}_{2}$ population was divided into two mixed samples each consisting of 30 lines, defined as $\mathrm{BC}_{2} \mathrm{~F}_{2} 6 \mathrm{P}^{+}$and $\mathrm{BC}_{2} \mathrm{~F}_{2} 6 \mathrm{P}^{-}$. All samples were snap-frozen in liquid nitrogen and ground into powder. The total RNA of each sample was extracted using TRIzol Reagent (Invitrogen, Carlsbad, CA, USA) according to the manufacturer's recommendations. The quantity and integrity of the total RNA were assessed using an Agilent 2100 Bioanalyzer (Agilent Technologies, PaloAlto, CA, USA) and 1\% agarose gel electrophoresis. Only total RNA samples with RIN values $\geq 8$ were used for constructing the cDNA libraries.

\section{Illumina and PacBio RNA-Seq library construction and sequencing}

Following the protocol of the Gene Expression Sample Prep Kit (Illumina, San Diego, CA, USA), a total of 15 libraries, namely, 11 libraries from $A$. cristatum leaves, stems and roots (3 biological replicates) and unfertilized caryopses (2 biological replicates) and 4 libraries from Fukuho, Pubing 3035, $\mathrm{BC}_{2} \mathrm{~F}_{2} 6 \mathrm{P}^{+}$and $\mathrm{BC}_{2} \mathrm{~F}_{2} 6 \mathrm{P}^{-}$mixed RNA from leaves and caryopses from four different periods (no biological replicate), were constructed following the protocol of the Gene Expression Sample Prep Kit (Illumina, San Diego, CA, USA). Then, the 15 libraries were sequenced by Novogene Corporation (Beijing, China) 
using the Illumina HiSeq 2500 platform with a paired-end read length of $150 \mathrm{bp}$.

To develop a comprehensive catalogue of transcript isoforms, equal amounts of the total RNA from each sample of A. cristatum Z559 were pooled into a single sample and used for PacBio library preparation. Library preparation and sequencing were performed according to the PacBio Iso-Seq protocol by Novogene Corporation (Beijing, China). Two SMRT cells were run on the PacBio sequel platform with non-size-selected RNA from the mixed sample.

Raw PacBio SMRT sequences and Illumina RNA-Seq data for this study have been deposited in the NCBI under BioProject accession number PRJNA534411.

\section{Subread processing and error correction}

Briefly, each sequencing run was processed by $c c s$ (https://github.com/PacificBiosciences/ccs) to generate one representative circular consensus sequence (CCS) for each zero-mode waveguide (ZMW). Only ZMWs with at least one full pass (at least one subread with SMRT adapter on both ends) were used for the subsequent analysis. The CCSs were processed to remove primers and unwanted combinations, and sequences were oriented to the $5^{\prime}-3^{\prime}$ direction using lima (https:// github.com/pacificbiosciences/barcoding), which offers a specialized isoseq mode. Then, to create FLNC transcripts, poly(A) tails were trimmed and artificial concatemers were removed by refine in IsoSeq3 (https://github. com/PacificBiosciences/IsoSeq3). The FLNC transcripts were then clustered together using cluster. The final polishing step created a consensus sequence for each clustered transcript using arrow model in polish. BUSCO [36] was used to explore completeness according to conserved orthologue content.

\section{Functional annotation of FLNC transcripts of $A$. cristatum}

Trinotate was used for automatic functional annotation of FLNC transcripts. Trinotate uses a number of different wellreferenced methods for functional annotation, including homology search to known sequence data (SwissProt, release 2019_03), protein domain identification (Pfam 32.0) [37], protein signal peptide (signalP version 4, https:/www.cbs.dtu.dk/ cgi-bin/nph-sw_request?signalp), rRNA (RNAMMER, https:// www.cbs.dtu.dk/cgi-bin/sw_request?rnammer) and transmembrane domain (tmHMM version 3.2.1, https://www.cbs. dtu.dk/cgi-bin/nph-sw_request?tmhmm) prediction, and leveraging various annotation databases (eggNOG/GO/Kegg) [38]. The sequence with the best hit was considered the optimal annotation. All functional annotation data derived from the analysis of transcripts was integrated into a SQLite database; SQLite allows terms with specific qualities related to a desired scientific hypothesis to be searched quickly and efficiently and provides a means to create a whole annotation report for a transcriptome (https://github.com/Trinotate/
Trinotate.github.io). PLEK (version 1.2), which is a predictor of long non-coding RNAs and messenger RNAs based on kmer scheme and the support vector machine (SVM) algorithm, was used to distinguish long non-coding RNAs (lncRNAs) from messenger RNAs (mRNAs) [39].

\section{Analysis of tissue-enriched transcripts}

All raw sequence reads from the Illumina sequencing platform were cleaned by removing the RNA adapters and trimming the low-quality bases $(\mathrm{Q}<20)$ with a minimum read length of 36 bases using Trimmomatic (version 0.39) [40]. The cleaned reads of all samples from A. cristatum Z559 were mapped to FLNC transcripts using Bowtie2 (version 2.3.5) [41]. The proportion of transcripts with zero coverage and unmapped reads that were not mapped to the transcriptome were counted and used to evaluate the quality of the transcriptome. The fragments per kilobase of transcript per million mapped reads (FPKM) values of the transcripts were calculated using RSEM (version 1.3.1) [42]. "Expressed" transcripts were defined as those with both (1) an average FPKM greater than 4 and (2) a FPKM greater than 2 for each replicate of the given tissue [29]. Significantly differentially expressed transcripts within different tissues were identified using DESeq2 software with a false discovery rate $(\mathrm{FDR})<0.01$ and a different expression level $\log _{2}$ (Fold Change) $\geq 2$ (version 3.8) [43]. "Expressed" transcripts that were also significantly differentially expressed in a particular tissue compared to all other tissues were considered tissue-enriched transcripts. The Bioconductor package GOseq (version 3.8) was used to explore functional enrichment among the transcript sets showing tissue-specific expression. Gene Ontology (GO) terms with padj < 0.05 (hypergeometric test) and clusters were plotted using REVIGO [44].

\section{Comparison of FLNC transcripts of $A$. cristatum and wheat gene model}

A. cristatum FLNC transcripts were aligned and mapped with GMAP (version 2015-09-29) to the Chinese Spring International Wheat Genome Sequencing Consortium (IWGSC) RefSeq V1.0 reference sequences [45]. Only FLNC transcripts mapping to a single location were retained. Each FLNC transcript mapped to the wheat genome was compared with the existing gene models of IWGSC RefSeq v1.0 annotation by cuffcompare [46]. Transcripts that aligned to intergenic regions of the wheat genome were considered novel transcripts compared with wheat, and transcripts that could not be aligned to the wheat genome were considered $A$. cristatum-specific transcripts. The visualization of the distribution of FLNC transcripts over the IWGSC genome was performed using Circos software (version 0.69-6) [47]. 
Discovery of $A$. cristatum-specific genes in the wheat- $A$. cristatum translocation line Pubing $\mathbf{3 0 3 5}$

The $A$. cristatum FLNC transcripts, transcripts assembled using short read sequencing [21] and IWGSC wheat RefSeq V1.0 reference sequences [45] were integrated as the reference sequences in this study. To reduce redundancy, the sequences were clustered using CD-HIT-EST with sequence identity set to $100 \%$. Illumina RNA-Seq clean reads from Fukuho, Pubing 3035, $\mathrm{BC}_{2} \mathrm{~F}_{2}{ }_{6} \mathrm{P}^{+}$and $\mathrm{BC}_{2} \mathrm{~F}_{2} 6 \mathrm{P}^{-}$were aligned and mapped to the reference sequences using the STAR tool (version 2.7) [48], using the 2-pass STAR method with a minimum intron length of $20 \mathrm{bp}$, a maximum intron length of $20 \mathrm{~kb}$ and default settings for the other parameters. A raw count matrix containing Pubing 3035, $\mathrm{BC}_{2} \mathrm{~F}_{2} \_6 \mathrm{P}^{+}$, Fukuho and $\mathrm{BC}_{2} \mathrm{~F}_{2}-6 \mathrm{P}^{-}$was constructed using the featureCounts program [49]. Significant differences in the read counts of transcripts between translocation lines (Pubing 3035 and $\mathrm{BC}_{2} \mathrm{~F}_{2} 6 \mathrm{P}^{+}$) and nontranslocation lines (Fukuho and $\mathrm{BC}_{2} \mathrm{~F}_{2}{ }_{6} 6 \mathrm{P}^{-}$) were detected by the package DESeq2 [43]. The output of DESeq2 consisted of the transcript IDs, base mean values, $\log _{2}$ (fold change) for translocation versus non-translocation, standard error (IfcSE) values, Wald statistic values, Wald test $P$ values and adjusted $P$ values. The transcripts from $A$. cristatum, including FLNC and Trinity-assembled transcripts, that were found to have a $\log _{2}$ (fold change) $\leq-4$ and adjusted $\mathrm{P}$ value $\leq 0.05$ were considered to be from the translocation fragment of Pubing 3035. The transcripts from the translocation fragment of Pubing 3035 were used to search the IWGSC Chinese Spring annotation to find homologous genes for polymorphic marker development. BatchPrimer3 was used to design primer pairs [50]. PCR amplification was carried out on the DNA of A cristatum Z559, Pubing 3035 and Fukuho. PCR products were separated in 8\% non-denaturing polyacrylamide gels, visualized by silver staining and photographed.

\section{Results}

Construction and annotation of the FLNC transcriptome database for $A$. cristatum

After quality control, a total of $11,966,252$ subreads, namely, 6,447,695 and 5,518,557 subreads from two different cells, were successfully generated (Table 1). A total of 504,811 representative CCSs for ZMWs were obtained. A total of 405,302 CCSs were classified as FL transcripts based on the presence of $5^{\prime}$ primers, $3^{\prime}$ primers and poly(A) tails. After demultiplexing, refining, clustering and polishing of FL transcripts were performed, a total of 44,372 FLNC transcripts with a maximum length of $9468 \mathrm{bp}$, a N50 of $3572 \mathrm{bp}$ and average FL coverage of 5.1 were generated (Table 1 ). In addition, the proportion of incomplete transcripts of FLNC transcripts was only $6.30 \%$ in BUSCO analysis (Table 2). As expected, the PacBio FLNC transcripts were generally
Table 1 Statistics of different kinds of A. cristatum SMRT sequencing reads

\begin{tabular}{lll}
\hline Category & First cell & Second cell \\
\hline No. of subreads & $6,447,695$ & $5,518,557$ \\
No. of CCS & 260,305 & 244,506 \\
No. of FL transcripts & 208,321 & 196,981 \\
No. of FLNC transcripts & 201,518 & 190,834 \\
No. of FLNC transcripts after merged & 392,352 & \\
No. of FLNC transcripts after clustered & 44,372 & \\
and polished & & \\
Average full-length coverage & 5.1 & \\
Maximum FLNC reads length (bp) & 9468 & \\
Average transcript length (bp) & 1874 & \\
N50 length (bp) & 3572 & \\
\hline
\end{tabular}

Notes: CCS represents circular consensus sequence; FLNC represents full-length, non-concatemer

longer and more complete than the transcripts assembled via the Illumina sequencing platform in previous studies [20, 21] (Fig. 2; Table 2). However, the higher proportion of unmapped reads $(72.24 \%)$ indicated that PacBio could not detect all transcripts due to insufficient sequencing data (Table 2). These results indicated that the PacBio FLNCs and transcripts assembled by 2nd generation sequencing should be integrated to obtain a high-quality $A$. cristatum transcriptome database.

Functional annotation of the FLNC transcripts was conducted using 5 different public databases (Table 3; Fig. 3). Of these, 30,854 FLNC transcripts were found to have homologs in the SwissProt database. A total of 24, 588 transcripts had significant matches in the eggNOG database, and 23,996 transcripts received Pfam domain assignments. Furthermore, 23,754 transcripts had matches in the Kegg database, and 29,424 transcripts were associated with GO terms. Moreover, the numbers of FLNC transcripts with transmembrane regions, signal peptides and rRNA transcripts were 5601, 2344 and 329, respectively. Altogether, 32,318 FLNC transcripts had at least one annotation (Table 3 ). In addition to proteincoding RNAs, 8202 candidate non-coding RNAs were predicted in non-annotated FLNC transcripts.

\section{Tissue-enriched FLNC isoforms}

To analyse tissue-enriched transcript expression, a total of 11 transcriptome libraries were generated from 4 different tissues with multiple biological replicates of $A$. cristatum (Additional file 1: Table S1). The Illumina sequencing generated approximately 15 million sequencing reads in each sample. After filtering the low-quality reads, about 99.98\% of the sequencing reads were retained for downstream analysis. Quality-controlled RNA-Seq reads from the leaves, stems, roots and caryopses of $A$. cristatum were 
Table 2 Statistical comparison of transcriptome assembled by different sequencing platforms

\begin{tabular}{|c|c|c|c|c|}
\hline & Proportion of non-existing transcripts ${ }^{c}$ & Proportion of unmapped reads ${ }^{d}$ & BUSCO analysis with fragment ratio ${ }^{\mathrm{e}}$ & N50 (bp) \\
\hline Illumina_1 $1^{\text {a }}$ & $7.35 \%$ & $18.28 \%$ & $31.10 \%$ & 1026 \\
\hline Illumina_2 $2^{\mathrm{b}}$ & $23.3 \%$ & $48.02 \%$ & $8.80 \%$ & 651 \\
\hline PacBio & $13.67 \%$ & $72.24 \%$ & $6.30 \%$ & 3572 \\
\hline
\end{tabular}

Notes: ${ }^{a}$ represents transcripts assembled by Zhang [20] using the Illumina GAll sequencing platform; b represents transcripts assembled by Zhou [20] using the Illumina HiSeq 2500 sequencing platform; ${ }^{c}$ represents the proportion of transcripts with zero coverage after realignment of reads on the transcriptome; ${ }^{d}$ represents the proportion of unmapped reads that were not mapped to the transcriptome; ${ }^{\mathrm{e}}$ represents the proportion of incomplete transcripts in the BUSCO analysis

mapped to FLNC transcripts (Additional file 1: Table S1). "Expressed" transcripts were defined as those with both (1) an average FPKM greater than 4 and (2) an FPKM greater than 2 for each replicate of the given tissue [29], resulting in the detection of 12,251 leaf, 13,440 stem, 14, 192 root and 15,253 caryopsis protein-coding transcripts and 8899 transcripts that may have "housekeeping" functions and were expressed in all sampled tissues (Fig. 4a). As expected, GO enrichment analysis showed that basic cell biological and metabolic processes were enriched in the 8899 ubiquitously expressed transcript set, including terms such as organonitrogen compound metabolic and biosynthetic process, organic substance metabolism, protein and peptide metabolism, and amide metabolic and biosynthetic based process (Fig. 4b; Additional file 2: Table S2). Additionally, the ubiquitous category shared intracellular part, organelle, ribonucleoprotein complex, and mitochondrial part terms.

Tissue-enriched transcripts, that is, transcripts expressed at significantly higher levels in a particular tissue compared to all other tissues (FDR $\leq 0.01$, Fold Change $\geq 4$, FPKM $\geq 2$ ) were next identified in each type of tissue. We observed that the caryopsis tissue had the highest number of tissueenriched transcripts (1515), followed by leaf (266), root (210), and stem (32) tissues. As expected, GO analysis showed that tissue-enriched FLNC transcripts were

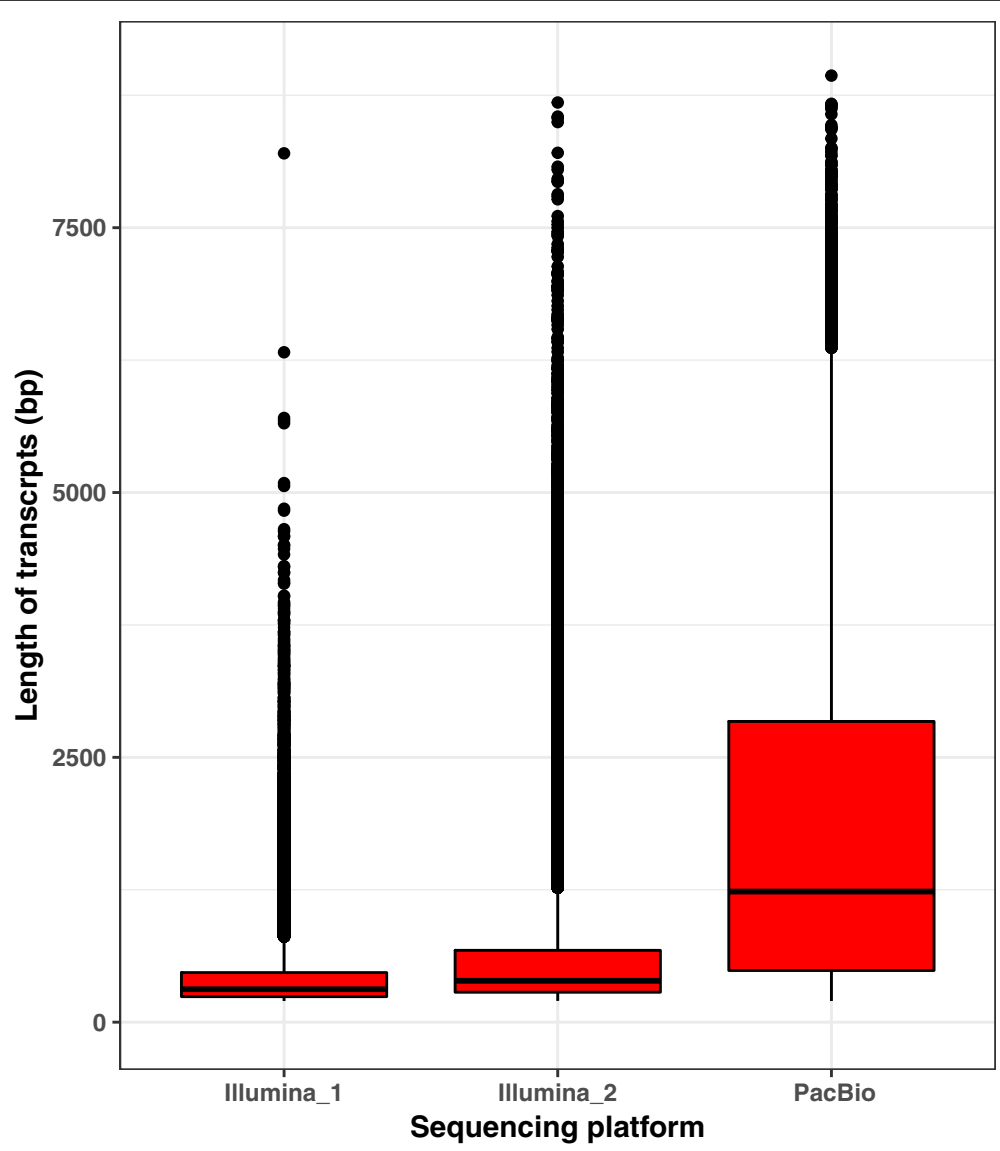

Fig. 2 Length distribution of transcripts obtained by different sequencing platforms. Illumina_1 and Illumina_2 represent the transcripts assembled by Zhang [20] and Zhou [21], respectively, using the Illumina sequencing platform 
Table 3 Statistics on functional annotations of the A. cristatum FLNC transcripts

\begin{tabular}{lll}
\hline Category & No. & Ratio \\
\hline FLNC transcripts & 44,372 & $100.0 \%$ \\
FLNC transcripts with blast hits to SwissProt & 30,854 & $69.5 \%$ \\
FLNC transcripts with blast hits to eggNOG & 24,588 & $55.4 \%$ \\
FLNC transcripts with blast hits to Pfam & 23,996 & $54.1 \%$ \\
FLNC transcripts with blast hits to Kegg & 23,754 & $53.5 \%$ \\
FLNC transcripts with GO terms & 29,424 & $66.3 \%$ \\
FLNC transcripts with transmembrane regions & 5601 & $12.6 \%$ \\
FLNC transcripts with signal peptides & 2344 & $5.3 \%$ \\
FLNC transcripts with rRNA transcripts & 329 & $0.7 \%$ \\
FLNC transcripts with at least one annotation & 32,318 & $72.8 \%$ \\
FLNC transcripts with non-coding sequences & 8202 & $18.5 \%$ \\
\hline
\end{tabular}

enriched for particular molecular functions that varies with tissues. Leaf tissue-enriched transcripts were associated with photosynthesis, with GO terms such as oxidoreductase activity, ribulose-bisphosphate carboxylase activity, photosynthesis dark reaction, carbon-carbon lyase activity, chloroplast, and flavonoid biosynthetic process. (Fig. 4c; Additional file 3: Table S3). In addition, the stem tissueenriched set was associated with many well-characterized transporter activity functions, including transferase activity, transferring glycosyl groups, transferring hexosyl groups, sucrose 1F-fructosyltransferase activity, fructosyltransferase activity, peptide:proton symporter activity, solute:proton symporter activity, solute:cation symporter activity, amide transmembrane transporter activity, symporter activity, and proton-dependent peptide secondary active transmembrane transporter activity GO terms (Fig. 4d; Additional file 4: Table S4). GO enriched analysis of the root tissue suggested that, in addition to expected categories associated with response to stress (response to external biotic stimulus, response to fungus, and response to biotic stimulus, regulation of defence response to fungus, and regulation of response to stimulus) and signal transduction (hormonemediated signalling pathway, salicylic acid mediated signalling pathway, ethylene-activated signalling pathway and phosphorelay signal transduction system), response to chitin, oxygen-containing compound, and organonitrogen compound terms appeared in the root-enriched transcript

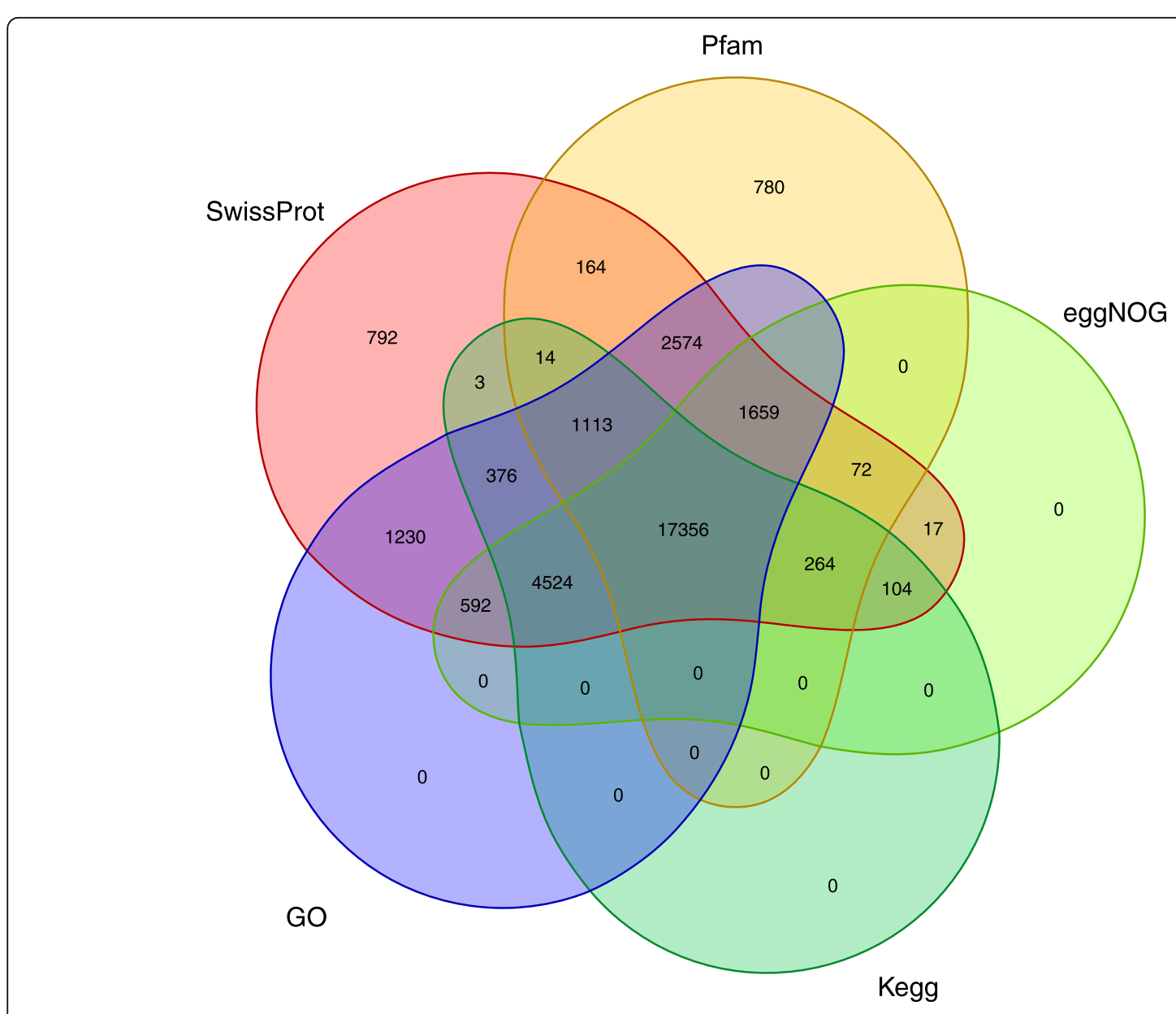

Fig. 3 Venn diagram showing the overlap of Pfam, SwissProt, eggNOG, GO and Kegg annotations of A. cristatum FLNC transcripts 

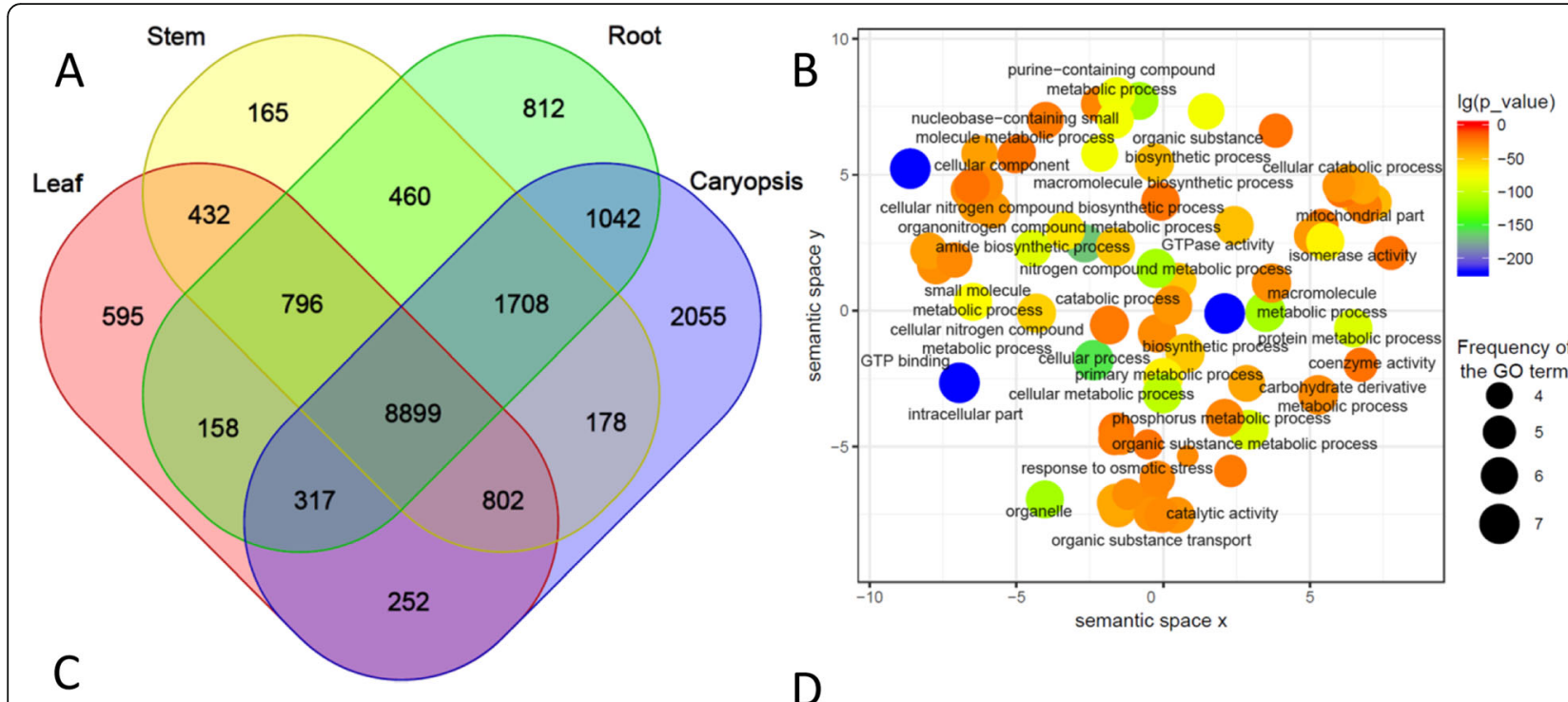

D
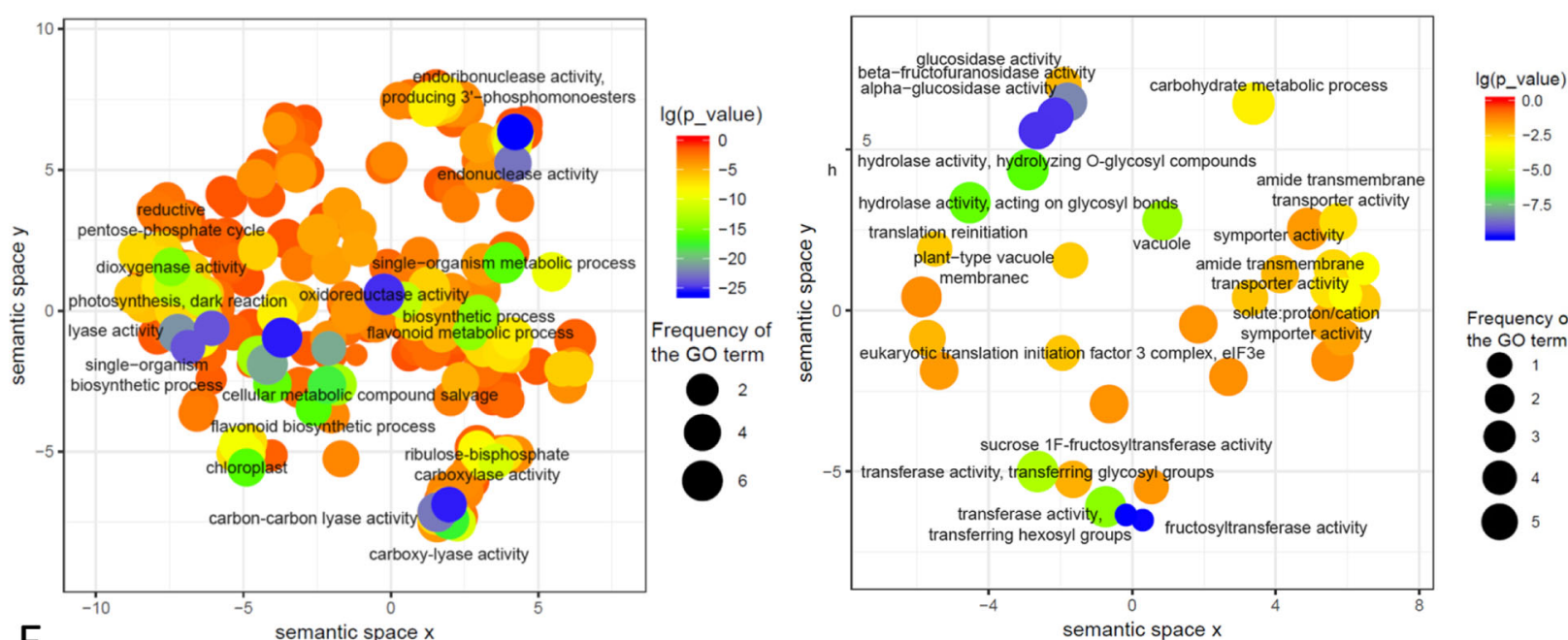

E

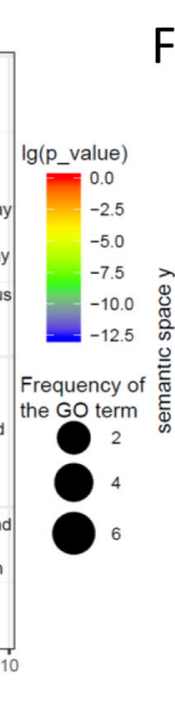

F
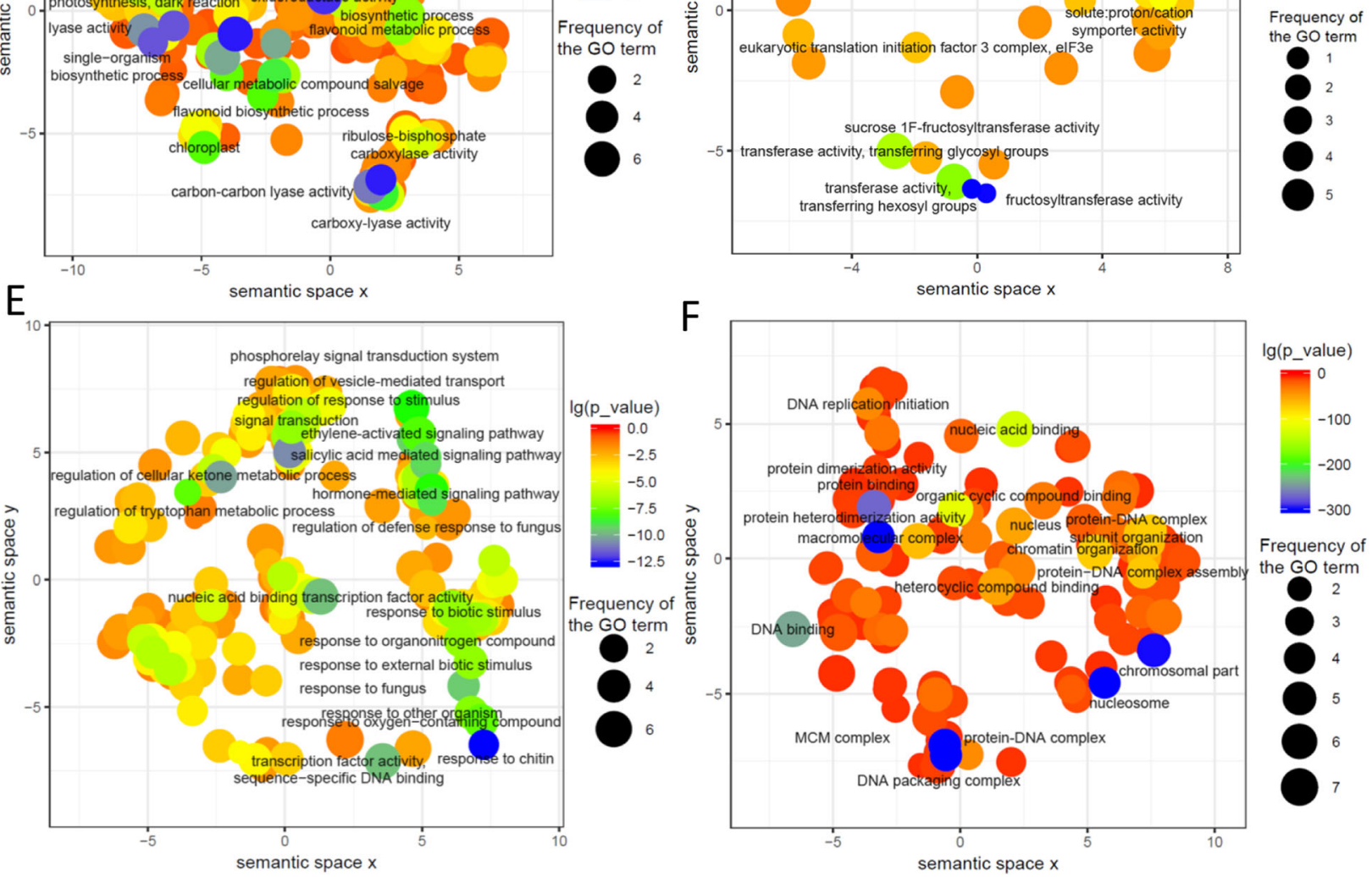

g(p_value)

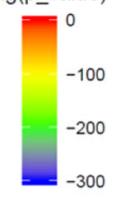

Frequency of the GO term

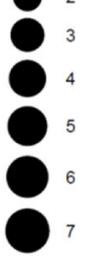

Fig. 4 (See legend on next page.) 
(See figure on previous page.)

Fig. 4 Tissue expression analysis of A. cristatum FLNC transcripts. a, Number of the protein-coding transcripts expressed in each sampled tissue. b, GO enrichment analysis for transcripts expressed in all sampled tissues. c, GO enrichment analysis for transcripts enriched in leaves. $\mathbf{d}$, GO enrichment analysis for transcripts enriched in stems. e, GO enrichment analysis for transcripts enriched in roots. f, GO enrichment analysis for transcripts enriched in caryopses

list (Fig. 4e; Additional file 5: Table S5). The vast majority of GO terms associated with the caryopsis tissue-enriched genes were related to cellular processes, including protein heterodimerization activity, protein-DNA complex, DNA packaging complex, nucleosome, chromosomal part, protein dimerization activity, and DNA/nucleic acid/protein binding/heterocyclic compound binding terms (Fig. 4f; Additional file 6: Table S6). In summary, these tissueenriched GO terms may provide insight into gene expression in A. cristatum tissue development and maintenance.

\section{FLNC transcripts compared with wheat gene model}

To compare transcripts between A. cristatum and wheat, the 44,372 FLNC transcripts were aligned to the IWGSC RefSeq v1.0 genome (Fig. 5) and compared with the wheat gene set model. A total of 43,020 FLNC transcripts were mapped to 17,510 loci that were spread across the wheat genome (Fig. 5b and c). Among these transcripts, 16,374 FLNC transcripts had multiple exons, and 4604 loci had multiple transcripts, with an average of 1.8 transcripts per locus. The distribution and density of FLNC transcripts on the wheat genome were calculated for all chromosomes in the wheat genome, and sharply decreased from the telomeres to centromeres in the whole wheat chromosomal regions (Fig. 5b). The number of FLNCs in each chromosome was not directly proportional to the chromosomal length and gene number. The most FLNC transcripts were aligned to the homologous group 2 chromosomes, whereas the homologous group 6 chromosomes contained the fewest FLNC transcripts (Fig. 5e). Interestingly, the highest FLNC transcript number and density were observed on the wheat D genome (17,326, 43.5 FLNC transcripts/10 Mb), followed by the wheat B genome (13,500, 25.8 FLNC transcripts/10 Mb), and the wheat A genome (11,612, 23.4 FLNC transcripts/10 Mb) (Fig. 5b, e and f). The distribution and density of the wheat-genome loci to which FLNC transcripts were mapped were similar to the distribution and density of FLNC transcripts (Fig. 5c, g and h). In total, 3398 novel FLNC transcripts were mapped to the intergenic regions of the wheat genome that did not overlap with wheat genes (Fig. 5d and i). The density of the novel transcripts also decreased from the chromosome ends towards the
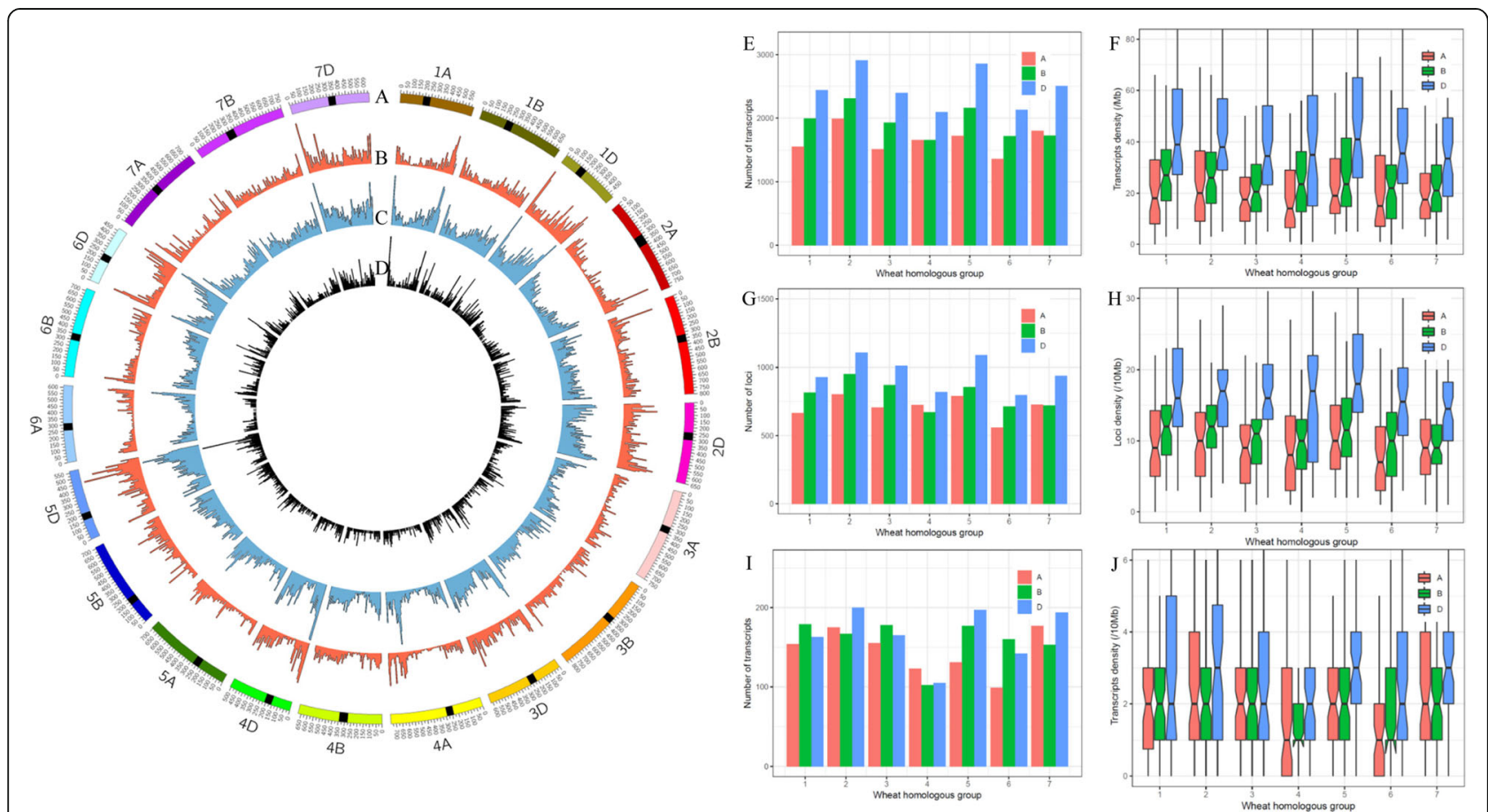

Fig. 5 Distribution of orthologues of A. cristatum transcripts in the wheat genome. a, Kanyotype of the wheat genome. The putative pericentromeric-centromeric regions are marked with black [51]. b, A. cristatum FLNC transcript density distribution; transcript density was calculated in a 10-Mb window. c, Loci density distribution; loci density was calculated in a 10-Mb window. d, Novel transcript density distribution; transcript density was calculated in a 10-Mb window. $\mathbf{e}$, Distribution of FLNC transcripts in the wheat genome. $\mathbf{f}$, Box plot of transcript density in the wheat genome. $\mathbf{g}$, Distribution of loci in the wheat genome. $\mathbf{h}$, Box plot of loci density in the wheat genome. i, Distribution of novel transcripts in the wheat genome. $\mathbf{j}$, Box plot of novel transcript density in the wheat genome 
centromeres (Fig. 5d), and the highest density was also observed in the wheat $\mathrm{D}$ genome (Fig. 5j).

GO analysis showed that novel FLNC transcripts were enriched for nucleic acid biological activity and biosynthetic processes, such as DNA polymerase activity, endonuclease activity, DNA recombination, integration and DNA biosynthetic processes, RNA-DNA hybrid ribonuclease activity, and nucleotidyltransferase activity (Fig. 6a; Additional file 7: Table S7). In addition, there were 1352 FLNC transcripts that were not aligned to the wheat genome that are considered to be $A$. cristatum-specific transcripts compared with wheat. The vast majority of GO terms associated with the A. cristatum-specific transcripts, including the COPI vesicle coat, retrograde vesicle-mediated transport from Golgi to endoplasmic reticulum, and Golgi vesicle transport terms, were related to protein transport processes in the cytoplasm. Additionally, these transcript categories shared terms associated with multi-organism metabolic processes such as the RNA-DNA hybrid ribonuclease activity, transporter activity of nucleobase:cation symporter, uptake transmembrane and nucleobase transmembrane terms (Fig. 6b; Additional file 8: Table S8). Thus, these 4750 FLNC transcripts, containing 3398 novel and 1352 A. cristatum-specific transcripts, might represent particularly positive selection compared with wheat and be helpful for understanding the genetic diversity of Triticeae.

\section{Identification of candidate genes associated with} thousand-grain weight in $A$. cristatum-wheat translocation line Pubing 3035

A total of 4 transcriptome libraries were generated from mixed RNA from the leaves and caryopses of Fukuho, Pubing $3035, \mathrm{BC}_{2} \mathrm{~F}_{2} 6 \mathrm{P}^{+}$and $\mathrm{BC}_{2} \mathrm{~F}_{2} 6 \mathrm{P}^{-}$sampled during four different periods. Illumina sequencing generated 42 ,
700,222, 30,258,610, 29,705,108 and 30,538,203 sequence reads in Fukuho, Pubing 3035, $\mathrm{BC}_{2} \mathrm{~F}_{2} 6 \mathrm{P}^{+}$and $\mathrm{BC}_{2} \mathrm{~F}_{2}$ $6 \mathrm{P}^{-}$, respectively. After filtering the low-quality reads, approximately $99.98 \%$ of the sequencing reads $(42,692$, 045 reads for Fukuho, 30,253,410 reads for Pubing 3035, $29,699,108$ reads for $\mathrm{BC}_{2} \mathrm{~F}_{2}{ }_{6} \mathrm{P}^{+}$and $30,533,230$ for $\left.\mathrm{BC}_{2} \mathrm{~F}_{2} 6 \mathrm{P}^{-}\right)$were retained for downstream analysis (Additional file 1: Table S1).

To identify genes specifically expressed in the translocation fragment, high-quality clean sequencing data were aligned to the reference sequences from an integration of the $A$. cristatum transcriptome and the wheat genome. Differential analysis using DESeq2 revealed that a total of $12 \mathrm{~A}$. cristatum transcripts exhibited differential expression between non-translocation and translocation lines that met the parameters of $\log _{2}($ fold change $) \leq-4$ and adjusted $P$ value $\leq 0.05$ (Table 4). The sequences of these 12 significantly differentially expressed transcripts were used as queries to search orthologous regions from genome sequences of wheat; the search indicated that homologous genes were located in the same interval on chromosome $6 \mathrm{~A} / \mathrm{B} / \mathrm{D}$. These intervals ranged from the TraesCS6A02G191200 gene to the TraesCS6A02G202900 gene on chromosome 6A, spanning $82.8 \mathrm{Mbp}$, from the TraesCS6B02G219700 gene to the TraesCS6B02G233700 gene on chromosome 6B, spanning $80.9 \mathrm{Mbp}$, and from the TraesCS6D02G174400 gene to the TraesCS6D02G187400 gene on chromosome 6D, spanning 88.7 Mbp (Additional file 9: Table S9). transcript/24685 and TRINITY_DN94508_c0_g1_i1, transcript/16718 and TRINITY_DN118140_c0_g2_i2, transcript/14210 and transcript/ 9968 and TRINITY_DN12662_c0_g1_i1 and TRINITY_ DN75295_c0_g1_i1 corresponded to the same homologs of the wheat genome, suggesting that they might be isoforms of the same gene or be derived from different homologous
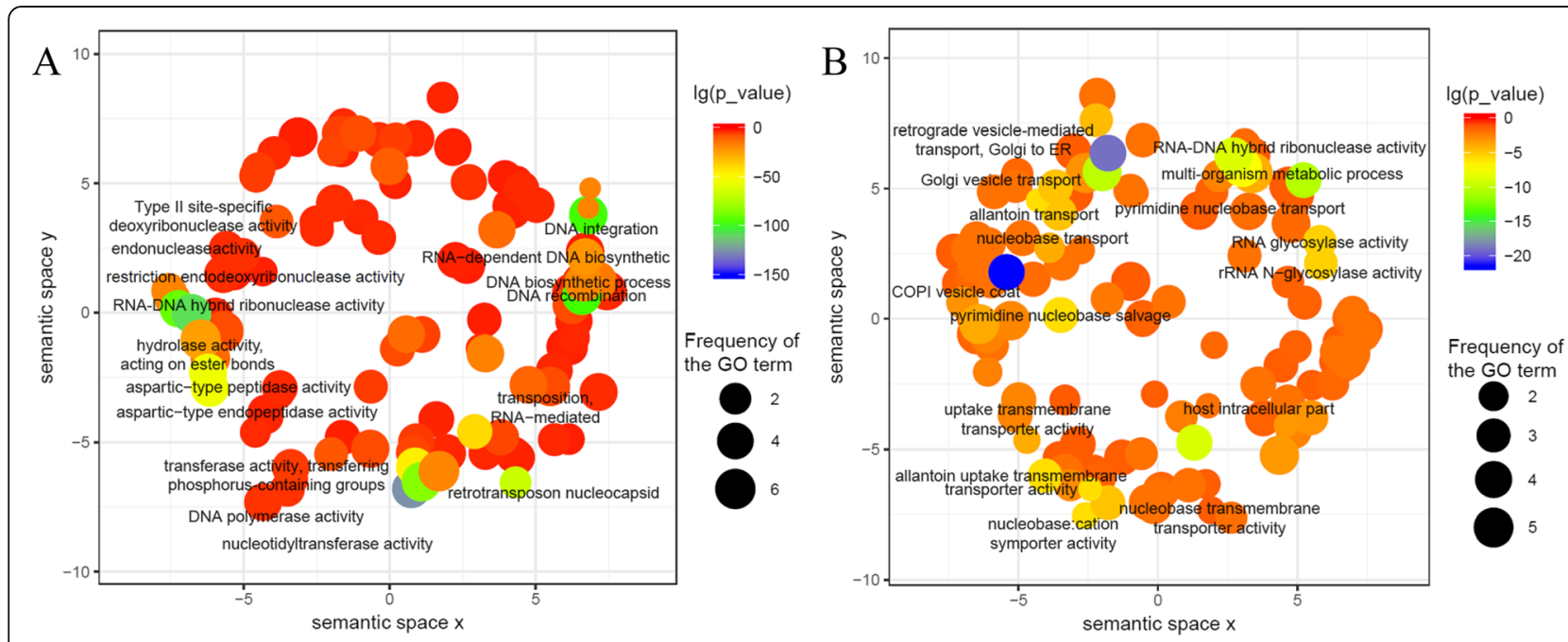

Fig. $6 \mathrm{GO}$ enrichment analysis for novel and A. cristatum-specific transcripts. a, GO enrichment analysis for novel transcripts. b, GO enrichment analysis for A. cristatum-specific transcripts 
Table 4 Statistics of the analysis of 6P translocation fragment-specific transcripts in Pubing 3035

\begin{tabular}{|c|c|c|c|c|c|c|c|c|c|c|}
\hline Transcript ID & $\begin{array}{l}\text { Fukuho }\left(6 \mathrm{P}^{-}\right) \\
\text {read count }\end{array}$ & $\begin{array}{l}\mathrm{BC}_{2} \mathrm{~F}_{2} 6 \mathrm{P}^{-} \\
\text {read count }\end{array}$ & $\begin{array}{l}\mathrm{BC}_{2} \mathrm{~F}_{2} 6 \mathrm{P}^{+} \\
\text {read count }\end{array}$ & $\begin{array}{l}\text { Pubing } 3035(6 \mathrm{P}+) \\
\text { read count }\end{array}$ & $\log _{2} F C^{a}$ & IfCSE ${ }^{b}$ & stat $^{c}$ & Pvalue $^{d}$ & padje & $\begin{array}{l}\text { Polymorphic } \\
\text { markers }\end{array}$ \\
\hline transcript/2610 & 0 & 2 & 86 & 127 & -7.04 & 1.18 & -5.95 & $2.62 \mathrm{E}-09$ & $2.30 \mathrm{E}-05$ & WGRG7 \\
\hline transcript/24685 & 1 & 2 & 691 & 372 & -8.79 & 0.96 & -9.12 & $7.52 \mathrm{E}-20$ & $1.55 \mathrm{E}-15$ & WGRG8 \\
\hline TRINITY_DN94508_c0_g1_i1 & 0 & 1 & 74 & 48 & -7.25 & 1.58 & -4.59 & 4.42E-06 & 9.73E-03 & WGRG8 \\
\hline transcript/7882 & 0 & 0 & 27 & 26 & -7.49 & 1.77 & -4.23 & 2.31E-05 & $3.84 \mathrm{E}-02$ & WGRG9 \\
\hline transcript/29056 & 0 & 0 & 28 & 47 & -7.99 & 1.71 & -4.67 & 3.03E-06 & 8.11E-03 & WGRG10 \\
\hline transcript/34773 & 0 & 0 & 144 & 174 & -10.08 & 1.53 & -6.59 & $4.29 \mathrm{E}-11$ & 5.29E-07 & WGRG11 \\
\hline transcript/16718 & 1 & 1 & 34 & 56 & -5.82 & 1.28 & -4.53 & 5.87E-06 & 1.17E-02 & WGRG12 \\
\hline TRINITY_DN118140_C0_g2_i2 & 0 & 0 & 64 & 118 & -9.27 & 1.60 & -5.80 & $6.65 \mathrm{E}-09$ & 3.73E-05 & WGRG12 \\
\hline transcript/14210 & 0 & 0 & 69 & 119 & -9.32 & 1.59 & -5.86 & 4.50E-09 & 3.47E-05 & WGRG13 \\
\hline transcript/9968 & 0 & 0 & 30 & 39 & -7.87 & 1.71 & -4.60 & $4.16 \mathrm{E}-06$ & 9.49E-03 & WGRG13 \\
\hline TRINITY_DN12662_CO_g1_i1 & 0 & 0 & 64 & 87 & -9.00 & 1.59 & -5.66 & $1.49 \mathrm{E}-08$ & 7.09E-05 & WGRG15 \\
\hline TRINITY_DN75295_c0_g1_i1 & 0 & 1 & 52 & 60 & -7.13 & 1.57 & -4.53 & 5.85E-06 & 1.17E-02 & WGRG15 \\
\hline
\end{tabular}

Notes: ${ }^{a}$ represents $\log _{2}\left(\right.$ fold change) for translocation lines (Pubing 3035 and $\mathrm{BC}_{2} \mathrm{~F}_{2} 6 \mathrm{P}^{+}$) versus non-translocation lines $\left(\right.$Fukuho and $\left.\mathrm{BC}_{2} \mathrm{~F}_{2}{ }^{6} \mathrm{P}^{-}\right) ;{ }^{\mathrm{b}}$ represents standard error; ${ }^{C}$ represents Wald statistic; ${ }^{d}$ represents Wald test $P$ value; ${ }^{\text {e }}$ represents adjusted $P$ values

genes (Additional file 9: Table S9). We developed polymorphic markers based on the sequences of homologous genes in the wheat $6 \mathrm{~A} / \mathrm{B} / \mathrm{D}$ chromosomal regions corresponding to the 12 differentially expressed transcripts (Tables 4; Additional file 10: Table S10; Additional file 11: Figure S1). The orthologous genomic regions of the translocation fragment in A. cristatum were identified in wheat chromosome 6A (Fig. 7), indicating that the wheat chromosome interval corresponding to the A. cristatum translocation fragment in Pubing 3035 was from the TraesCS6A02G190200 to the TraesCS6A02G204000 gene of chromosome $6 \mathrm{~A}$ and that obvious rearrangements could be observed on the $6 \mathrm{P}$ translocation segment compared with the wheat 6A chromosome (Fig. 7). According to these results, it could be speculated that the genomic region of the translocation fragment in A. cristatum shows collinearity with chromosomes $6 \mathrm{~A}$ of wheat.

The functions of these 12 significantly differentially expressed transcripts were investigated and one of them, transcript/7882, was homologous to the rice gene OsUBP15/LG1, which encodes a constitutively expressed ubiquitin-specific protease 15 (OsUBP15) that possesses deubiquitination activity in vitro and is a positive regulator of grain width and size in rice [52]. The marker WGRG9 was developed from transcript/7882 and corresponding with TraesCS6A02G192600, TraesCS6B02G231700 and TraesCS6D02G179700 (Fig. 7; Additional file 9: Table S9). Therefore, the orthologue of WGRG9 in the corresponding $\mathrm{P}$ genomic region can serve as a candidate gene for control of thousand-grain weight in Pubing 3035; this gene should be subjected to functional verification in a future study.

\section{Discussion}

To broaden the wheat gene pool and provide new potential genes for wheat breeding, many desirable genes from A. cristatum have been transferred into common wheat by the intergeneric hybridization of common wheat and A. cristatum [53]. However, the progress in A. cristatum genomic studies lags far behind the production and application of wheat $-A$. cristatum derivatives, limiting our understanding of the genetic effects of alien fragments/ genes on agronomic traits and the application of these derivative lines to wheat breeding projects [54]; one of the main reasons for this lack of understanding is that the reference genome of $A$. cristatum is not currently available because of its large size and high heterozygosity. Therefore, the construction of a FL cDNA sequence database for A. cristatum is critically important to fully unveil the molecular mechanisms of alien genes in wheat- $A$. cristatum novel germplasms until the wholegenome sequencing of $A$. cristatum has been achieved. In this study, a FL transcriptome database of A. cristatum was constructed using PacBio single-molecule technology, which provided useful information and showed three major features, which are described as follows.

Integration of PacBio FLNCs and transcripts assembled by 2nd generation sequencing in complex de novo transcriptome analysis

High-throughput transcriptome sequencing has recently become a popular technique because it is cost-effective, does not rely on a reference genome and can contribute to transcriptional analysis, molecular marker development and gene discovery [19]. In a previous study, de novo transcriptome assembly and unigene functional annotation 


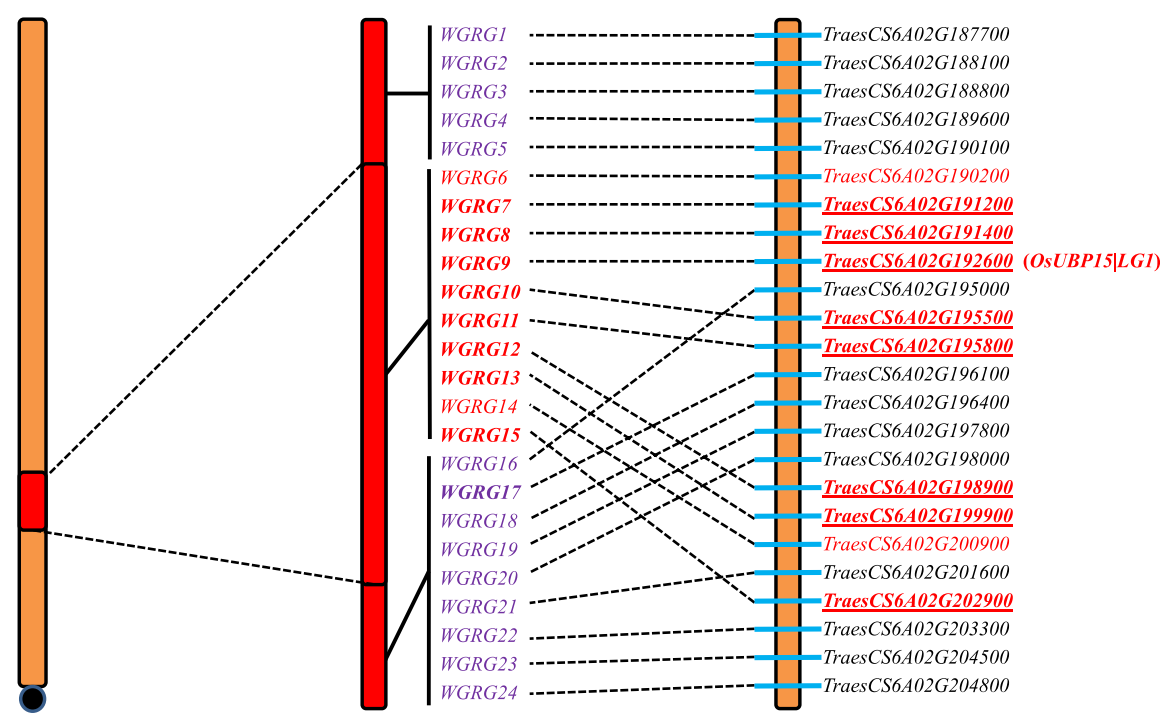

1A chromosome of Pubing 3035 6P chromosome of A. cristatum 6A chromosome of Chinese Spring

Fig. 7 Comparative genomics map between the 6P chromosome translocation fragment in Pubing 3035 and the 6A chromosome of wheat

were conducted in A. cristatum with the Illumina sequencing technique, and gene resources that were related to traits of interest and specific to A. cristatum within the tribe Triticeae, as well as the phylogenetic relationship and interspecific variation between $A$. cristatum and wheat, were identified $[20,21]$. However, transcriptome analyses in species without reference genome sequences often encounter complicated problems, especially in the assembly of sequencing reads; thus, the assembly and annotation of A. cristatum is incomplete and error-prone (Table 2), which severely impedes in-depth molecular breeding and gene functional studies of A cristatum. Compared to Illumina RNA-Seq, PacBio SMRT-Seq has the ability to assemble FL transcripts due to its longer read length and zero need for PCR amplification during library construction. Therefore, we analysed mixed samples of leaves, stems, roots and caryopses with single-molecule long-read sequencing technology from PacBio and reported the first FL transcriptome dataset of $A$. cristatum comprising 44,372 FLNC transcripts. Compared with the assembled transcripts of $A$. cristatum obtained from the Illumina sequence platform in previous studies, the average length and integrity of the transcripts in this study were greatly increased (Fig. 2; Table 2). However, the higher proportion of unmapped reads indicated that PacBio could not detect all transcripts due to insufficient sequencing data (Table 2). Therefore, PacBio FLNCs and transcripts assembled by 2nd generation sequencing should be integrated to obtain a high-quality A. cristatum transcriptome database in complex de novo transcriptome analysis. The integrated transcriptome database will provide resources for the study of gene expression and the discovery and development of specific markers in $A$. cristatum.

\section{Tissue-enriched transcript expression and enrichment analysis of $A$. cristatum}

The study of tissue-specific genes will provide insights into tissue development and evolution and has been verified in several plant species. MacMillan used cotton as a model to study different secondary cell walls and the expression of the genes involved in their formation via RNA deep sequencing of the stem and seed fibre, revealing the subtleties of the gene regulation underlying the diversity of plant secondary cell walls [55]. To comprehensively annotate genes in the yerba mate phenylpropanoid pathway and to evaluate differential expression profiles, Fay generated tissue specific transcriptomic profiles [56]. The combination of reference sequences for FLNC transcriptomes and RNA-Seq technology allowed the identification of tissue-specific expressed transcripts of $A$. cristatum. A comprehensive transcriptome profile of the major tissue types comprising the ovary and young fruit of tomato was obtained using laser-capture microdissection and RNA-Seq, revealing great diversity in gene expression associated with tissue type and developmental stage [57]. In this study, we performed RNA-Seq profiling of gene expression in four tissues of $A$. cristatum. By mapping the FLNC transcripts in this study, we identified tissue-specific expressed transcripts and enriched GO terms using statistical and gene set enrichment methods. A total of 266, 210, 32 and 1515 tissue-enriched transcripts were identified in leaves, roots, stems and caryopses, respectively. As expected, GO analysis showed that tissueenriched FLNC transcripts were enriched for particular 
molecular functions that varies with tissues. Leaf tissueenriched genes were associated with photosynthesis (Fig. 4c; Additional file 3: Table S3). The stem tissue-enriched set was associated with many well-characterized transporter activity functions (Fig. 4d; Additional file 4: Table S4). GO enrichment analysis suggested that in addition to expected categories associated with responses to stress and signal transduction, terms associated with responses to chitin, oxygen-containing compounds, and organonitrogen compounds appeared in the root-enriched transcripts list (Fig. 4e; Additional file 5: Table S5). The vast majority of GO terms associated with the caryopsis tissue-enriched genes were related to cellular processes (Fig. 4f; Additional file 6: Table S6). The isolation and RNA-Seq analysis of four major tissues of $A$. cristatum revealed that the transcriptomes of tissues differ from one another, highlighting the importance of transcriptionally profiling specific developmental tissues to understand the corresponding biology. These data provide resources to explore the application of A. cristatum genes in wheat breeding.

\section{An effective strategy for excavating functional candidate genes from wheat and wild relative-derived germplasms expressing a given trait}

For many decades, desirable traits have been transferred from wild relatives into wheat through a series of wide crossings by cytogeneticists and breeders. However, because of the suppressed recombination between the alien chromatin and the domesticated chromatin, alien introgressions, which are an important source of genetic variation in wheat breeding, have fallen into disfavour with many breeders due to the co-introduction of undesirable alleles of genes on the alien introgression, a process known as linkage drag [58]. Therefore, the desirable genes related to the target traits should be separated from the linked sequences when introducing alleles between wheat and wild relatives. Advanced biotechnologies, such as next-generation sequencing and homology-based cloning, have proven beneficial in accelerating gene discovery directly from derived lines of wheat and wild relatives $[16,59,60]$. However, most of these studies related to the cloning of genes from derived lines have focused on disease-resistant genes, and no relevant studies have reported the cloning of genes associated with complex traits such as yield directly from derived lines. Thus, new strategies need to be developed to fully access and exploit the rich gene source found in the wild relatives of wheat under a wheat background. In this study, by integrating transcriptome databases of a wild wheat relative, $A$. cristatum, and wheat genome sequences into a comprehensive reference sequence, we effectively excavated functional candidate genes from their translocation line. Furthermore, we reported the successful application of this strategy in the excavation of wild relative-specific genes from the wheat and A. cristatum-derived translocation line Pubing
3035, in which the alien chromosome fragment has a positive regulatory effect on thousand-grain weight and spike length in wheat [15]. A total of 12 A. cristatum transcripts were identified as differentially expressed and verified by PCR experiments between the non-translocation and translocation lines, and their homologous genes were located in the same interval on chromosome 6A/B/D. Previous studies have shown that the $\mathrm{P}$ genome is more closely related to wheat genome A than to the B and D genomes. Therefore, a comparative genomics map between the 6P chromosome fragment in Pubing 3035 and the wheat 6A chromosome was constructed by developing polymorphic molecular markers of conserved homologous genes between the 6P and $6 \mathrm{~A}$ chromosomes. The results show that A. cristatum transcript/7882 corresponds with TraesCS6A02G192600, TraesCS6B02G231700 and TraesCS6D02G179700 (Fig. 7; Additional file 9: Table S9) and is homologous to rice gene OsUBP15/LG1, which encodes a constitutively expressed ubiquitin-specific protease 15 (OsUBP15) that possesses de-ubiquitination activity in vitro and is a positive regulator of grain width and size in rice [52], suggesting that their orthologue in the corresponding $\mathrm{P}$ genomic region could serve as a candidate gene for controlling thousand-grain weight in Pubing 3035. The effective research method used in this study can be applied in other studies to discover candidate genes in wheat and wild relative-derived translocation lines with prominent traits.

\section{Conclusion}

Single-molecule long-read transcriptome sequencing of A. cristatum Z559 was performed using the PacBio Sequel platform. A total of 44,372 FLNC transcripts were constructed and annotated. Tissue-enriched FLNC transcripts were revealed in A. cristatum using RNA-Seq. Then, novel and $A$. cristatum-specific transcripts were identified by comparison with the wheat gene model set. Furthermore, by integrating the $A$. cristatum transcripts with the wheat genome as a reference sequence, 12 candidate $A$. cristatum transcripts associated with thousandgrain weight were identified in Pubing 3035 and verified to be genuine via polymorphic molecular markers. The present study not only provides comprehensive transcriptomic insights and information for A. cristatum but also proposes a new method for the exploration of functional genes from wheat relatives under a wheat genetic background.

\section{Supplementary information}

Supplementary information accompanies this paper at https://doi.org/10. 1186/s12864-019-6416-4.

Additional file 1: Table S1. Summary of the Illumina sequencing data. Additional file 2: Table S2. GO enrichment analysis for transcripts expressed in all sampled tissues. 
Additional file 3: Table S3. GO enrichment analysis for transcripts enriched in leaves.

Additional file 4: Table S4. GO enrichment analysis for transcripts enriched in stems.

Additional file 5: Table S5. GO enrichment analysis for transcripts enriched in roots.

Additional file 6: Table S6. GO enrichment analysis for transcripts enriched in caryopses.

Additional file 7: Table S7. GO enrichment analysis for novel transcripts.

Additional file 8: Table S8. GO enrichment analysis for A. cristatumspecific transcripts.

Additional file 9: Table S9. Homologous wheat genes of 6P translocation fragment-specific transcripts in Pubing 3035

Additional file 10: Table S10. Polymorphic molecular marker primer sequences developed between translocation and non-translocation lines.

Additional file 11: Figure S1. PCR amplification patterns of polymorphic markers. The red arrows show A. cristatum-specific DNA fragments. $M$ is a DNA marker; Lane 1 is A. cristatum Z559; Lane 2 is Pubing 3035; Lane 3 is Fukuho.

\section{Abbreviations}

Bgt: Blumeria graminis f. sp. tritici; CCS: Circular consensus sequence; FDR: False discovery rate; FL: Full-length; FLNC: Full-length, non-concatemer; FPKM: Fragments per kilobase of transcript per million mapped reads: Fukuho: Fukuhokumugi; GO: Gene Ontology; IWGSC: International Wheat Genome Sequencing Consortium; IncRNAs: Long non-coding RNAs; mRNAs: Messenger RNAs; PacBio: Pacific Biosciences; RNA-Seq: RNA sequencing; SMRT: Single-molecule, real-time; SVM: Support vector machine; ZMW: Zero-mode waveguide

\section{Acknowledgements}

Not applicable.

\section{Authors' contributions}

SZ, JZ1 and LL conceived and designed the experiments. SZ, JZ2, and HM performed the experiments. $\mathrm{HH}, J Z 2, Z Z, X Y$ and $X L$ contributed to the material planting and sample collection. SZ contributed to data analysis. SZ, $Y L$ and $W L$ wrote the manuscript. All authors discussed the results and commented on the manuscript. All authors read and approved the final manuscript.

\section{Funding}

This project is supported by grants from the National Science Foundation of China (31801359) and China Agriculture Research System (CARS-03). Apart from providing funds, they were not involved in the study design, data collection, analysis, interpretation, or manuscript writing.

\section{Availability of data and materials}

The sequence data have been deposited in the NCBI under BioProject accession number PRJNA534411.

\section{Ethics approval and consent to participate}

Not applicable.

\section{Consent for publication}

Not applicable.

\section{Competing interests}

The authors declare that they have no competing interests.

\section{Author details}

${ }^{1}$ National Key Facility for Crop Gene Resources and Genetic Improvement, Institute of Crop Sciences, Chinese Academy of Agricultural Sciences, Beijing 100081, China. ${ }^{2}$ Rice Research Institute, Guangdong Academy of Agricultural Sciences, Guangzhou 510640, China.
Received: 2 May 2019 Accepted: 19 December 2019

Published online: 27 December 2019

\section{References}

1. Food and Agriculture Organization of the United Nations. FAOSTAT statistics database, Food balance sheets. 2017. http://www.fao.org/faostat/en/\#data/FBS.

2. Charmet G. Wheat domestication: lessons for the future. C R Biol. 2011;334: 212-20.

3. Khoury CK, Bjorkman AD, Dempewolf H, Ramirez-Villegas J, Guarino L, Jarvis A, Rieseberg LH, Struik PC. Increasing homogeneity in global food supplies and the implications for food security. P Natl Acad Sci USA. 2014;111:4001-6.

4. White J, Law J, MacKay I, Chalmers K, Smith J, Kilian A, Powell W. The genetic diversity of UK, US and Australian cultivars of Triticum aestivum measured by DArT markers and considered by genome. Theor Appl Genet. 2008;116:439-53.

5. Friebe B, Jiang J, Raupp WJ, Mclntosh RA, Gill BS. Characterization of wheatalien translocations conferring resistance to diseases and pests: current status. Euphytica. 1996;91:59-87.

6. Jauhar PP, Chibbar RN. Chromosome-mediated and direct gene transfers in wheat. Genome. 1999:42:570-83.

7. Qi LL, Friebe B, Zhang P, Gill BS. Homoeologous recombination, chromosome engineering and crop improvement. Chromosom Res. 2007;15:3-19.

8. Przewieslik-Allen AM, Burridge AJ, Wilkinson PA, Winfield MO, Shaw DS, McAusland L, King J, King IP, Edwards KJ, Barker GLA. Developing a highthroughput SNP-based marker system to facilitate the introgression of traits from Aegilops species into bread wheat (Triticum aestivum). Front Plant Sci. 2019;9:1993.

9. Wang M, Wang S, Liang Z, Shi W, Gao C, Xia G. From genetic stock to genome editing: gene exploitation in wheat. Trends Biotechnol. 2018;36: 160-72.

10. Dewey DR. The genomic system of classification as a guide to intergeneric hybridization with the perennial Triticeae. In: Gustafson JP, editor. Gene manipulation in plant improvement. Boston: Springer; 1984. p. 209-79.

11. Li L, Dong Y, Zhang R, Li X, Li P. Cytogenetics and self-fertility of hybrids between Triticum aestivum L. and Agropyron cristatum (L.) Gaertn. Acta Genet Sin. 1995:22:109-14

12. Zhang Z, Song L, Han H, Zhou S, Zhang J, Yang X, Li X, Liu W, Li L. Physical localization of a locus from Agropyron cristatum conferring resistance to stripe rust in common wheat. Int J Mol Sci. 2017;18:2403.

13. Pan C, Li Q, Lu Y, Zhang J, Yang X, Li X, Li L, Liu W. Chromosomal localization of genes conferring desirable agronomic traits from Agropyron cristatum chromosome 1P. PLOS One. 2017:12:e0175265.

14. Zhang J, Zhang JP, Liu WH, Wu XY, Yang XM, Li XQ, Lu YQ, Li LH. An intercalary translocation from Agropyron cristatum 6P chromosome into common wheat confers enhanced kernel number per spike. Planta. 2016;244:853-64.

15. Zhang J, Zhang JP, Liu WH, Han HM, Lu YQ, Yang XM, Li XQ, Li LH. Introgression of Agropyron cristatum 6P chromosome segment into common wheat for enhanced thousand-grain weight and spike length. Theor Appl Genet. 2015:128:1827-37.

16. Xing L, Hu P, Liu J, Witek K, Zhou S, Xu J, Zhou W, Gao L, Huang Z, Zhang R. Pm21 from Haynaldia villosa encodes a CC-NBS-LRR that confers powdery mildew resistance in wheat. Mol Plant. 2018. https://doi.org/10.1016/j.molp. 2018.02.013.

17. He H, Zhu S, Zhao R, Jiang Z, Ji Y, Ji J, Qiu D, Li H, Bie T. Pm21, encoding a typical CC-NBS-LRR protein, confers broad-spectrum resistance to wheat powdery mildew disease. Mol Plant. 2018. https://doi.org/10.1016/j.molp. 2018.03.004

18. Placido DF, Campbell MT, Folsom JJ, Cui X, Kruger GR, Baenziger PS, Walia $H$. Introgression of novel traits from a wild wheat relative improves drought adaptation in wheat. Plant Physiol. 2013;161:1806-19.

19. Meena S, Kumar SR, Venkata Rao DK, Dwivedi V, Shilpashree HB, Rastogi S, Shasany AK, Nagegowda DA, De Novo sequencing and analysis of lemongrass transcriptome provide first insights into the essential oil biosyntesis of aromatic grasses. Front Plant Sci. 2016;7

20. Zhang JP, Liu WH, Han HM, Song LQ, Bai L, Gao ZH, Zhang Y, Yang XM, Li $X Q$, Gao AN, Li LH. De novo transcriptome sequencing of Agropyron cristatum to identify available gene resources for the enhancement of wheat. Genomics. 2015;106:129-36.

21. Zhou SH, Yan BQ, Li F, Zhang J, Zhang J, Ma HH, Liu WH, Lu YQ, Yang XM Li XQ, Liu X, Li LH. RNA-Seq analysis provides the first insights into the phylogenetic relationship and interspecific variation between Agropyron cristatum and wheat. Front Plant Sci. 2017:8:1644. 
22. Zhu C, Li X, Zheng J. Transcriptome profiling using Illumina- and SMRTbased RNA-seq of hot pepper for in-depth understanding of genes involved in CMV infection. Gene. 2018;666:123-33.

23. Zhang B, Liu J, Wang $X$, Wei Z. Fulll-length RNA sequencing reveals unique transcriptome composition in bermudagrass. Plant Physiol Biochem. 2018;132:95-103.

24. Yi S, Zhou X, Li J, Zhang M, Luo S. Full-length transcriptome of Misgurnus anguillicaudatus provides insights into evolution of genus Misgurnus. Sci Rep. 2018;8:11699.

25. Wang M, Wang P, Liang F, Ye Z, Li J, Shen C, Pei L, Wang F, Hu J, Tu L, et al. A global survey of alternative splicing in allopolyploid cotton: landscape, complexity and regulation. New Phytol. 2018;217:163-78.

26. Minio A, Massonnet M, Vondras A, Figueroa-Balderas R, Blanco-Ulate B, Cantu D. Iso-Seq allows genome-independent transcriptome profiling of grape berry development. G3-Genes Genom Genet. 2019;9:3755-67.

27. Liu J, Jiang H, Zan J, Bao Y, Dong J, Xiong L, Nie L. Single-molecule longread transcriptome profiling of Platysternon megacephalum mitochondrial genome with gene rearrangement and control region duplication. RNA Biol. 2018;15:1244-9.

28. Kim J-A, Roy NS, Lee I-h, Choi A-Y, Choi B-S, Yu Y-S, N-i P, Park K-C. Kim S, Yang $\mathrm{H}-\mathrm{s}$, Choi I-Y. genome-wide transcriptome profiling of the medicinal plant Zanthoxylum planispinum using a single-molecule direct RNA sequencing approach. Genomics. 2018;111:973-9.

29. Kaletsky R, Yao V, Williams A, Runnels AM, Tadych A, Zhou S, Troyanskaya OG, Murphy CT. Transcriptome analysis of adult Caenorhabditis elegans cells reveals tissue-specific gene and isoform expression. PLoS Genet. 2018;14:e1007559.

30. Jia D, Wang Y, Liu Y, Hu J, Guo Y, Gao L, Ma R. SMRT sequencing of fulllength transcriptome of flea beetle Agasicles hygrophila (Selman and Vogt). Sci Rep. 2018;8:2197.

31. Hu P, Wang T, Liu H, Xu J, Wang L, Zhao P, Xing X. Full-length transcriptome and microRNA sequencing reveal the specific generegulation network of velvet antler in sika deer with extremely different velvet antler weight. Mol Gen Genomics. 2019;294:431-43.

32. Deng Y, Zheng H, Yan Z, Liao D, Li C, Zhou J, Liao H. Full-length transcriptome survey and expression analysis of Cassia obtusifolia to discover putative genes related to aurantio-obtusin biosynthesis, seed formation and development, and stress response. Int J Mol Sci. 2018;19: 2476.

33. Chen X, Liu X, Zhu S, Tang S, Mei S, Chen J, Li S, Liu M, Gu Y, Dai Q, Liu T. Transcriptome-referenced association study of clove shape traits in garlic. DNA Res. 2018:25:587-96.

34. Chen J, Tang X, Ren C, Wei B, Wu Y, Wu Q, Pei J. Full-length transcriptome sequences and the identification of putative genes for flavonoid biosynthesis in safflower. BMC Genomics. 2018;19:548.

35. Zadoks JC, Chang TT, Konzak CF. A decimal code for the growth stages of cereals. Weed Res. 1974;14:415-21.

36. Simão FA, Waterhouse RM, loannidis $P$, Kriventseva EV, Zdobnov EM. BUSCO: assessing genome assembly and annotation completeness with single-copy orthologs. Bioinformatics. 2015;31:3210-2.

37. Finn RD, Bateman A, Clements J, Coggill P, Eberhardt RY, Eddy SR, Heger A, Hetherington K, Holm L, Mistry J, et al. Pfam: the protein families database. Nucleic Acids Res. 2014;42:D222-30

38. Tatusov RL, Fedorova ND, Jackson JD, Jacobs AR, Kiryutin B, Koonin EV, Krylov DM, Mazumder R, Mekhedov SL, Nikolskaya AN, Rao BS, Smirnov S, Sverdlov AV, Vasudevan S, Wolf YI, Yin JJ, Natale DA. The COG database: an updated version includes eukaryotes. BMC Bioinformatics. 2003;4:41.

39. Li A, Zhang J, Zhou Z. PLEK: a tool for predicting long non-coding RNAs and messenger RNAs based on an improved k-mer scheme. BMC Bioinformatics. 2014;15:311.

40. Bolger AM, Lohse M, Usadel B. Trimmomatic: a flexible trimmer for Illumina sequence data. Bioinformatics. 2014;30:2114-20.

41. Langmead B, Salzberg SL. Fast gapped-read alignment with bowtie 2. Nat Methods. 2012;9:357-9.

42. Li B, Dewey CN. RSEM: accurate transcript quantification from RNA-Seq data with or without a reference genome. BMC Bioinformatics. 2011;12:323.

43. Love MI, Huber W, Anders S. Moderated estimation of fold change and dispersion for RNA-seq data with DESeq2. Genome Biol. 2014;15:550.

44. Supek F, Bosnjak M, Skunca N, Smuc T. REVIGO summarizes and visualizes long lists of Gene Ontology terms. PloS One. 2011;6:e21800.

45. Appels R, Eversole K, Feuillet C, Keller B, Rogers J, Stein N, Pozniak CJ, Stein N, Choulet F, Distelfeld A, et al. Shifting the limits in wheat research and breeding using a fully annotated reference genome. Science. 2018;361:eaar7191.
46. Trapnell C, Roberts A, Goff L, Pertea G, Kim D, Kelley DR, Pimentel H, Salzberg SL, Rinn JL, Pachter L. Differential gene and transcript expression analysis of RNA-seq experiments with TopHat and cufflinks. Nat Protoc. 2012;7:562-78.

47. Krzywinski M, Schein J, Birol I, Connors J, Gascoyne R, Horsman D, Jones SJ, Marra MA. Circos: an information aesthetic for comparative genomics. Genome Res. 2009;19:1639-45.

48. Dobin A, Davis CA, Schlesinger F, Drenkow J, Zaleski C, Jha S, Batut P, Chaisson M, Gingeras TR. STAR: ultrafast universal RNA-seq aligner. Bioinformatics. 2013;29:15-21.

49. Liao Y, Smyth GK, Shi W. featureCounts: an efficient general purpose program for assigning sequence reads to genomic features. Bioinformatics. 2014;30:923-30.

50. You FM, Huo N, Gu YQ, Luo MC, Ma Y, Hane D, Lazo GR, Dvorak J, Anderson OD. BatchPrimer3: a high throughput web application for PCR and sequencing primer design. BMC Bioinformatics. 2008;9:253.

51. Su H, Liu Y, Liu C, Shi Q, Huang Y, Han F. Centromere satellite repeats have undergone rapid changes in polyploid wheat subgenomes. Plant Cell. 2019; 31:2035-51.

52. Shi C, Ren Y, Liu L, Wang F, Zhang H, Tian P, Pan T, Wang Y, Jing R, Liu T, et al. Ubiquitin specific protease 15 has an important role in regulating grain width and size in rice. Plant Physiol. 2019;180:381-91.

53. $L i L H$, Li XQ, Yang $X M$, Dong $Y C$, Chen XM. Introduction of desirable genes from Agropyron cristatum into common wheat by intergeneric hybridization. Sci Agric Sin. 1998;31:1-5 (in Chinese).

54. Zhou S, Zhang J, Che Y, Liu W, Lu Y, Yang X, Li X, Jia J, Liu X, Li L. Construction of Agropyron Gaertn. Genetic linkage maps using a wheat 660K SNP array reveals a homoeologous relationship with the wheat genome. Plant Biotechnol J. 2018;16:818-27.

55. MacMillan CP, Birke $H$, Chuah A, Brill E, Tsuji Y, Ralph J, Dennis ES, Llewellyn D, Pettolino FA. Tissue and cell-specific transcriptomes in cotton reveal the subtleties of gene regulation underlying the diversity of plant secondary cell walls. BMC Genomics. 2017;18:539.

56. Fay JV, Watkins CJ, Shrestha RK, Litwiñiuk SL, Talavera Stefani LN, Rojas CA, Argüelles CF, Ferreras JA, Caccamo M, Miretti MM. Yerba mate (llex paraguariensis, A. St.-Hil.) de novo transcriptome assembly based on tissue specific genomic expression profiles. BMC Genomics. 2018;19:891.

57. Pattison R, Csukasi F, Zheng Y, Fei Z, van der Knaap E, Catalá C. Comprehensive tissue-specific transcriptome analysis reveals distinct regulatory programs during early tomato fruit development. Plant Physiol. 2015;168:1684-701.

58. Hatta MAM, Steuernagel B, BBH W. Rapid gene cloning in wheat. In: Miedaner T, Korzun V, editors. Applications of genetic and genomic research in cereals. Einbeck: Woodhead; 2019. p. 65-95.

59. Cao $A H$, Xing LP, Wang XY, Yang XM, Wang $W$, Sun $Y L$, Qian $C$, Ni JL, Chen YP, Liu DJ, et al. Serine/threonine kinase gene Stpk-V, a key member of powdery mildew resistance gene Pm21, confers powdery mildew resistance in wheat. P Natl Acad Sci USA. 2011;108:7727-32.

60. Hurni S, Brunner S, Buchmann G, Herren G, Jordan T, Krukowski P, Wicker T, Yahiaoui N, Mago R, Keller B. Rye Pm8 and wheat Pm3 are orthologous genes and show evolutionary conservation of resistance function against powdery mildew. Plant J. 2013;76:957-69.

\section{Publisher's Note}

Springer Nature remains neutral with regard to jurisdictional claims in published maps and institutional affiliations.

\section{Ready to submit your research? Choose BMC and benefit from:}

- fast, convenient online submission

- thorough peer review by experienced researchers in your field

- rapid publication on acceptance

- support for research data, including large and complex data types

- gold Open Access which fosters wider collaboration and increased citations

- maximum visibility for your research: over $100 \mathrm{M}$ website views per year

At $\mathrm{BMC}$, research is always in progress.

Learn more biomedcentral.com/submissions 